\title{
Attentional Selection of Multiple Goal Positions Before Rapid Hand Movement Sequences: An Event-related Potential Study
}

\author{
Daniel Baldauf and Heiner Deubel
}

\begin{abstract}
A dot-probe paradigm was used to provide physiological evidence for the parallel selection of multiple movement goals before rapid hand movement sequences. Participants executed a sequence of manual pointing movements to two out of three possible goal positions. During movement preparation, a taskirrelevant visual transient (a dot probe) was flashed either at one of both movement goals, or at the third, movement-irrelevant
\end{abstract}

\section{INTRODUCTION}

Visual attention plays a crucial role in the selection of objects that are relevant for goal-directed actions. It has been assumed that attention leads to preferred visual processing, and thus provides the relevant spatial information about the targets of an intended movement to the motor system (Neumann, 1987). Allport (1987) referred to this prioritized processing of movementrelevant parts of the visual scene as "selection-for-action." The selection of certain parts of the visual field among surrounding distracters has been shown to facilitate the detection of stimuli (Posner, 1980), as well as to improve the recognition and identification of presented objects (Treisman \& Gelade, 1980). Also on a neuronal basis, the processes of covertly deploying spatial attention and movement preparation seem to share common circuits (e.g., Awh, Armstrong, \& Moore, 2006). According to the "premotor theory of visual attention" (Rizzolatti, Riggio, \& Sheliga, 1994), spatially selective attention, in general, is a consequence of activation in cortical areas that code space for the programming of goal-directed motor actions in so-called spatial pragmatic maps. As proposed by a number of authors, different spatial pragmatic maps in parietal regions are activated depending on the type of action that is to be performed, and depending on the effector system that is to be used for these actions (e.g., Andersen \& Buneo, 2002; Snyder, Batista, \& Andersen, 2000; Colby, 1998; Andersen, Snyder, Bradley, \& Xing, 1997; Kawashima et al., 1996; Jeannerod, 1994).

Ludwig-Maximilians-Universität, Munich, Germany location. The results revealed that the N1 component induced by the presentation of the dot was enhanced if the dot was flashed at one of the movement goals, indicating that both target positions were attended before the initialization of the movement sequence. A second experiment showed that movement-irrelevant locations between the movement goals were not attended, suggesting that attention splits into spatially distinct foci.

At a behavioral level, a variety of studies have shown that the intention to perform a certain movement causes a covert shift of visual attention to the goal location in advance to the movement initialization. This was demonstrated most convincingly for saccadic eye movements, where several empirical studies analyzed the deployment of visual attention during the preparation of eye movements (e.g., Schneider \& Deubel, 2002; Irwin \& Gordon, 1998; Deubel \& Schneider, 1996; Hoffman \& Subramaniam, 1995; Kowler, Anderson, Dosher, \& Blaser, 1995). These behavioral studies revealed evidence for superior processing of visual events at the intended target location, and support the hypothesis that attentional shifts toward the goal position of a planned saccade are triggered during the saccade preparation period. Deubel and Schneider (1996; see also Schneider \& Deubel, 2002), for example, showed that the discrimination accuracy was considerably higher when the discrimination target was presented at the saccade goal than when it was presented at adjoining positions. This preferential processing of the saccade target was found even when the participants knew the location of the discrimination target in advance, indicating that the coupling of visual attention and saccade target selection is mandatory.

Selection-for-action is not restricted to the control of oculomotor responses. Goals for other kinds of movements such as goal-directed reaches also imply attentional shifts during their preparation. A number of recent studies, indeed, indicated that the coupling of visual attention and action preparation is not limited to the eye movement system, but is a general mechanism and 
rather independent of the effector system used. Deubel, Schneider, and Paprotta (1998), for example, showed that when participants prepare a pointing movement to a location, perceptual processing is selectively enhanced at the movement goal, already before movement onset.

The studies reviewed so far investigated the deployment of visual attention before single movements to a single target. In everyday life, however, motor behavior is more complex and only rarely restricted to single targets. In a recent study of coordinated bimanual reaching movements, we (Baldauf \& Deubel, 2008b) showed that attention shifts in parallel to the intended points of application for the left and right hand. Under natural conditions, many actions are movement chains consisting of several sequential components. The subcomponents have to be linked appropriately in order to fluently perform the complex actions. In natural sequential tasks as hand washing (Pelz \& Canosa, 2001) or preparing a sandwich (Hayhoe, Shrivastava, Mruczek, \& Pelz, 2003), it has been demonstrated that human actors are planning their movements several steps ahead and often gather important visual information about future reaching goals in advance of execution by so called lookahead fixations. Mennie, Hayhoe, and Sullivan (2007) studied eye, hand, and head movements while subjects built models with wooden slats. The authors found that anticipatory look-aheads occurred before about 20\% of all reaching movements and improved subsequent visuomotor coordination. In order to perform fluently in a sequential movement task, it may be efficient to take subsequent movements into account for the execution of the impending movement. The question arises whether in such complex actions, composed of several sequential movements, the selective processing of relevant visual information is also sequential, such that processing of the second target would occur only after the first movement is completed. Ballard, Hayhoe, and Pelz (1995) called this a "just-in-time" strategy. Alternatively, in fast movement sequences, the amount of time that elapses between the first and the second movements may be too short to effectively process the subsequent goal. Some of the information processing that is relevant for the second movement part could take place already before the onset of the initial movement segment, simultaneously with the selection of the first goal. It may even be possible that all the single movement parts are assembled into one action plan for the entire sequence in advance. This would imply that all action-relevant targets are selectively processed in advance of movement onset in order to specify the necessary movement parameters (such as movement direction or amplitude).

Only very few studies have investigated the specific properties of attention deployment before sequential movements. For planning of manual actions, we (Baldauf, Wolf, \& Deubel, 2006) studied attentional deployment in rapid reaching sequences. As a primary task, participants had to perform fast double- or triple-pointing move- ments to various peripheral goal positions. Briefly, after a go-signal for the movement but before movement onset, small target letters were presented either at one of the movement goals or at other, movement-irrelevant positions. After completion of the movement sequence, the nonspeeded secondary task was to identify the target letter that had been presented during the movement preparation period. This secondary task served as a measure of the allocation of visual attention during sequence preparation. The results showed that the ability to identify target letters in the secondary task was superior at all goal locations of the planned sequence. From these findings, we concluded that in rapid reaching sequences, up to three target positions are selected in advance during the movement preparation interval before the first movement starts. Moreover, the analysis of intermediate locations that lay between the first and second goals of a double reach showed that attention splits into spatially distinct foci, which are selected in parallel.

The purpose of the present event-related potential (ERP) study was to provide convergent, electrophysiological evidence that multiple goal positions are attentionally selected when sequential hand movements are planned. In the present study, the allocation of attention prior to the movement initialization was examined with a dot-probe paradigm. Mangun and Hillyard (1988) were the first to introduce the dot-probe paradigm to study the effects of spatially selective visual attention. Essentially, this approach uses the amplitude of the neural response elicited by a probe stimulus as an indicator of how much processing resources were allocated to that location (see also Mangun \& Hillyard, 1990, 1991). The visual ERP was shown to be particularly sensitive to the direction of spatial attention. Since then, probe stimuli were used in various tasks, demonstrating that dot probes presented at attended locations elicit larger sensory-evoked P1/N1 components than stimuli at unattended locations (Luck \& Hillyard, 1995; Luck et al., 1994; Mangun, Hillyard, \& Luck, 1993).

\section{EXPERIMENT 1}

\section{Methods}

\section{Participants}

Nine students, aged between 23 and 28 years ( 4 men), participated in the experiment. All had normal or corrected-to-normal vision and were right-handed. They were paid for their participation and gave their informed consent in advance.

\section{Experimental Setup}

The participants sat in a dimly lit room. The stimuli were presented on a 21-in. color monitor with a frame frequency 
of $100 \mathrm{~Hz}$, providing a spatial resolution of $1024 \times$ 768 pixels. The active screen size was $40 \times 30 \mathrm{~cm}$; viewing distance was $58 \mathrm{~cm}$. Pointing movements were executed on a slightly inclined plane in front of the participant. A one-way mirror was adjusted in front of the subject such that the visual stimuli appeared to be projected onto the pointing plane. The mirror between the pointing plane and the participant's face avoided the occlusion of the visual stimuli by the hand or arm and also allowed hand movements without visual feedback about the position of the hand and fingers. The visual stimuli were presented on a gray background, which was adjusted to a mean luminance of $2.2 \mathrm{~cd} / \mathrm{m}^{2}$. The relatively moderate background brightness is important to minimize the effects of phosphor persistence (Wolf \& Deubel, 1997). The luminance of the visual stimuli was $23 \mathrm{~cd} / \mathrm{m}^{2}$.

Pointing movements were recorded with a Fastrak electromagnetic position and orientation measuring system (Polhemus Inc., 1993), consisting of a sender unit and a small receiver mounted on the tip of the index finger of the participant's right hand. The sender unit was fixed at a distance of $60 \mathrm{~cm}$ from the participant. The device had a spatial accuracy of $0.8 \mathrm{~cm}$. The frequency bandwidth of the system is $120 \mathrm{~Hz}$ and the signal delay is approximately $4 \mathrm{msec}$. In order to provide visual feedback about the spatial positions of the fingertip during an initial positioning period, a small red lightemitting diode (LED) controlled by the computer was attached to the sensor. Eye fixation was controlled by the electrooculogram (EOG). An adjustable chin rest helped to reduce head movements.

\section{Stimuli and Procedure}

Figure 1A shows the sequence of stimuli in a typical trial of Experiment 1. The gray screen contained a continuously visible fixation cross at its center. The participants were required to fixate at this central cross during the whole experimental block. At the beginning of each trial, they also positioned their right index finger at the central cross. After $400 \mathrm{msec}$, a stimulus configuration was presented consisting of three crosses in three of the four corners of the screen. The crosses appeared $5^{\circ}$ in the visual periphery and extended $1.2^{\circ}$ of visual angle. After a random delay of 650-1200 msec, an acoustic gosignal was given, which had a pitch of either 200 or $500 \mathrm{~Hz}$. The participants were instructed to perform a double-pointing sequence to two out of the three crosses of the configuration as soon as they heard the go-signal. If the go-signal was a low-frequency beep, the participants first pointed to the cross in the middle of the configuration and then went on to the next cross in the clockwise direction. If they heard a high-frequency beep, they first pointed to the cross in the middle and then immediately pointed to the cross in the counterclockwise direction. Participants were asked to execute the double-pointing sequence as fast and as fluently as possible. Speed and accuracy were equally stressed.

In order to determine the deployment of visual attention during the movement preparation period, a taskirrelevant dot probe was flashed at a delay of $150 \mathrm{msec}$ after the acoustic go-signal-well before the initialization of the pointing sequence-at the location of one of the peripheral crosses. The probe consisted of a circular disk with a diameter of $1.2^{\circ}$. The probe was presented for 70 msec superimposed on one of the peripheral crosses. After the execution of the required movement sequence, the participants received visual feedback about the pointing accuracy at the final goal position.

\section{Design}

Initially, each participant performed a training block consisting of 60 trials: These were not included in the data analysis. After this initial training, the participants performed five experimental blocks, each consisting of 120 trials. The critical factor that was varied in this experiment was the position where the dot probe was flashed relative to the instructed movement targets. This factor (probe position) had three levels: (1) The dot probe was flashed at the first movement target (condition "1st MT"), or (2) at the second movement target position (condition "2nd MT"), or (3) it appeared at the third cross of the configuration that was not a pointing goal in the present trial and therefore was movement-irrelevant ("irr"). There were four possible cross configurations corresponding to the four quadrants of the screen. The acoustical go-signal that also coded the direction for the second part of the movement (clockwise vs. counterclockwise) was either a highor a low-frequency beep. In total, this led to 24 different conditions ( 4 possible cross configurations $\times 2$ acoustical direction cues $\times 3$ relative probe positions). The conditions were selected at random in each trial. Each condition was repeated five times in an experimental block.

\section{Recordings}

Electroencephalogram (EEG) data were continuously recorded by a BrainAmp system (Brain Products, Munich, Germany) from $64 \mathrm{Ag} / \mathrm{AgCl}$ electrodes mounted in an elastic cap (EasyCap, FMS). The electrodes were positioned according to the international 10-10 system and referenced to $\mathrm{Cz}$. The vertical electrooculogram (vEOG) was recorded bipolarly from electrodes above and below the left eye. The horizontal electrooculogram (hEOG) was recorded bipolarly from the outer canthi of both eyes. Electrode impedances were kept below $5 \mathrm{k} \Omega$ and as equal as possible in all electrodes. The signals were amplified and filtered on-line using a 0.1-100 Hz band-pass filter; the digitalization rate was $500 \mathrm{~Hz}$. The recorded signals were then $40-\mathrm{Hz}$ low-pass filtered off-line. 
Figure 1. Experimental procedures. (A) In

Experiment 1, stimulus configuration was presented at the beginning of each trial consisting of three crosses. This triangular arrangement could be oriented to any of the four quadrants. After a random delay of 650-1200 msec, a high- or low-frequency beep was presented as an acoustical go-signal. Upon this tone, the participants were requested to perform a double-pointing sequence. The first movement had to be directed to the cross at the middle position of the virtual triangle. Dependent on the pitch of the go-signal, the second reach led to the next cross in either the clockwise (low-frequency beep) or the counterclockwise direction (high-frequency beep). At 150 msec after the onset of the go-signal, a dot probe was flashed for $70 \mathrm{msec}$ at one of the three cross locations. (B) In Experiment 2, the initial stimulus configuration consisted of five crosses that were equidistantly aligned on a virtual semicircle around the central fixation. Upon the go-signal, the participants were requested to perform a double-pointing sequence with the first movement part being directed to the cross at the middle position of the configuration and the second reach to the cross two positions further in either a clockwise or counterclockwise direction.

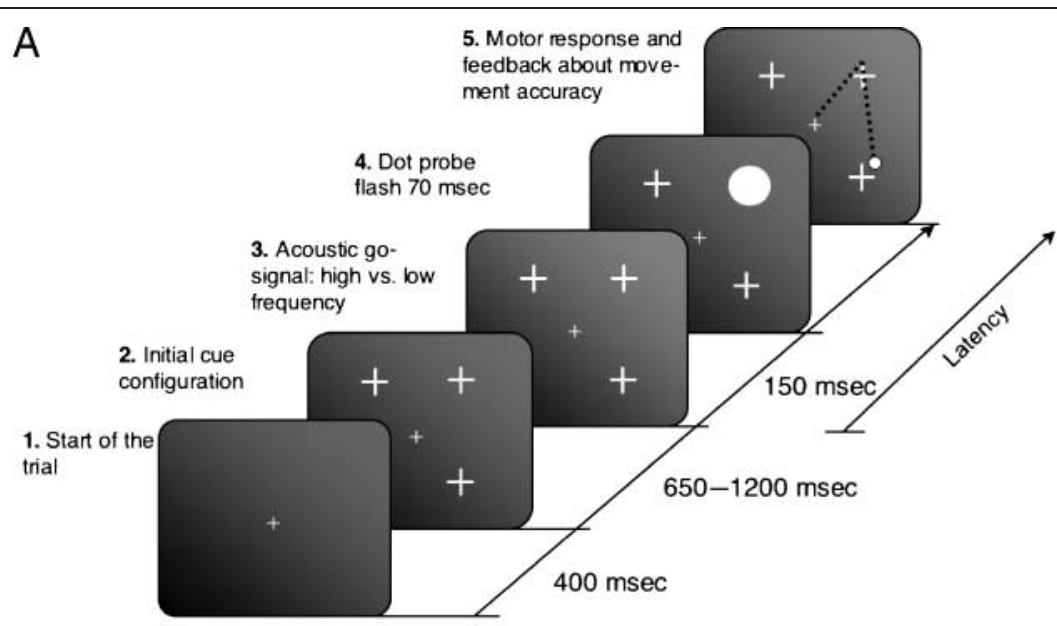

B

5. Motor response and feedback about movement accuracy

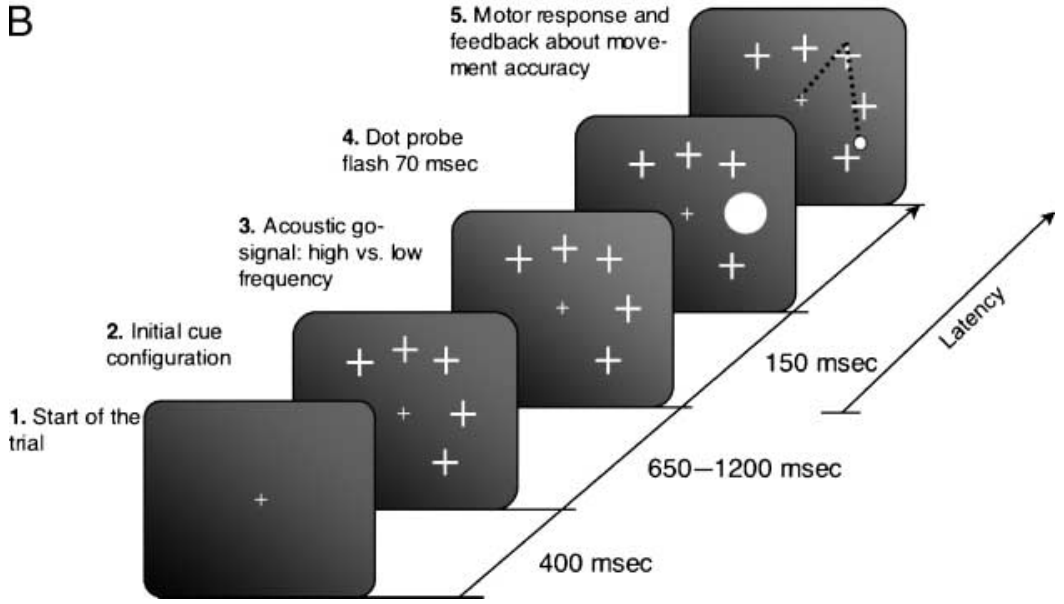

The continuous EEG data were segmented into predefined analysis windows of $1200 \mathrm{msec}$ duration, starting $200 \mathrm{msec}$ prior to and ending $1000 \mathrm{msec}$ after the presentation of the acoustical go-signal. Trials with eye blinks (defined by a voltage at Fpz exceeding $\pm 80 \mu \mathrm{V}$ ), with saccades (a voltage at hEOG or vEOG exceeding $\pm 80 \mu \mathrm{V}$ ), or with muscle artifacts (a voltage at any site exceeding $\pm 100 \mu \mathrm{V}$ ) were excluded from further analysis. After this rejection of artifacts, there was still some residual activity in the EOG channels that was caused by small eye movements counterbalancing the deviations in head position when the reach was initialized. We corrected the ERPs for these residual eye movements by applying the algorithm of Gratton, Coles, and Donchin (1983), which computes propagation factors that characterize the relationship between EOG and EEG traces.
Because the analysis of the EEG data revealed that the elicited ERPs were only very weakly lateralized, we computed separate ERP averages only for the three possible relative positions of the dot probe with respect to the actual pointing goals, irrespective of the quadrant in which the dot probe had appeared. The epochs were time-locked to the onset of the go-signal, and averages were computed relative to the 200-msec baseline before the onset of this imperative stimulus. Locking the evoked ERPs to the onset of the go-signal seems appropriate because movement preparation, hence selectionfor-action, are hypothesized to start at this point in time. A consequence of locking the ERPs to the go-signal is that the components elicited by the probe are shifted by the stimulus onset asynchrony (SOA) between go-signal and onset of the probe $(150 \mathrm{msec})$. The mean ERP amplitudes of the components that were elicited by dot 
probes appearing at the three different relative positions were analyzed in a repeated measures analysis of variance (ANOVA). The factors of this ANOVA were probe position ("1st MT," "2nd MT," or "irr") and electrode site (with the levels "O1," "O2," "P3," "P4," "C3," and "C4"). The analyses were based on the mean amplitude of the N1 component (relative to baseline) that was elicited by the onset of the dot probe. The statistical analyses were performed with the "R" statistical package (Ihaka \& Gentleman, 1996).

\section{Results}

Rejection of Trials due to Movement Errors

$5.4 \%$ of all trials were discarded because the instructed target was missed by more than $3^{\circ}$. In most of these errors, participants responded incorrectly to the acoustical direction cue and executed the second movement, for example, in counterclockwise instead of clockwise direction. $6.7 \%$ of all trials were rejected because of the occurrence of eye movements or other artifacts in the EEG (e.g., muscle activity). Another 6.9\% of trials with movement onset latencies longer than $600 \mathrm{msec}$ were also excluded from further analysis. Finally, because we wanted to ensure that the dot probe was presented only during the movement preparation period, we discarded $1.5 \%$ of trials with latencies shorter than $220 \mathrm{msec}$ (SOA of $150 \mathrm{msec}$ plus $70 \mathrm{msec}$ presentation time of the dot probe).

\section{Movement Performance}

Figure 2A illustrates some typical trajectories for one participant. Participants performed fast and accurately in the experimental blocks. The mean pointing error between the landing positions of the first movement and the center of the target item was $1.54^{\circ}\left(S E=0.15^{\circ}\right)$. Figure $2 \mathrm{~B}$ shows the landing positions of the first (circles) and the second movement parts (crosses) for all participants.

In a more detailed analysis, we calculated the directional error of the landing position at the first movement goals assigning deviations in clockwise direction with positive values and deviations in counterclockwise direction with negative values. We tested whether the endpoints of the first movement part systematically depended on the direction of the subsequent movement. In trials with clockwise sequence production, the mean directional error of the first movement component was $0.08^{\circ}\left(S E=0.02^{\circ}\right)$, and in counterclockwise trials was $0.10^{\circ}\left(S E=0.02^{\circ}\right)$. This difference was not significant $[t(8)=0.59, p>.50]$, indicating that the endpoints of the first movement component did not systematically depend on whether the sequence was planned to be continued in clockwise or in counterclockwise direction.

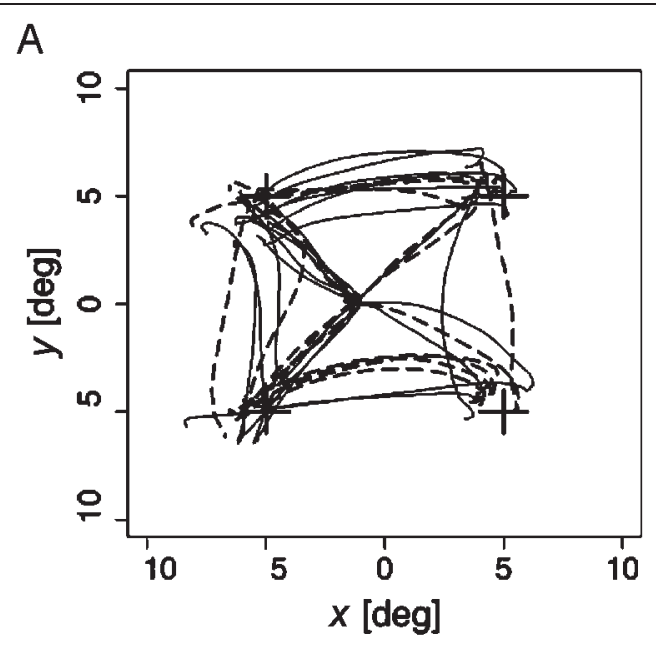

B

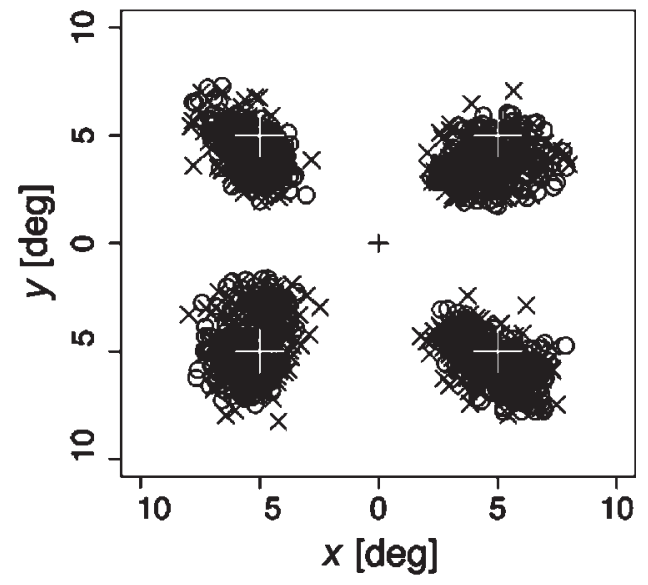

Figure 2. (A) Example movement trajectories from one participant in Experiment 1. The first movement of the double-pointing sequence was directed to one of the four corners. The second movement led to the next position either in a clockwise (solid lines) or in a counterclockwise (dashed lines) direction, depending on the acoustical go-signal. (B) Landing positions of the first (circles) and second (crosses) movements of the sequence.

The initial movement started with a mean latency of $371 \mathrm{msec}(S E=27.4 \mathrm{msec})$ after presentation of the acoustic cue and had an average duration of $201 \mathrm{msec}$ $(S E=14.7 \mathrm{msec})$. The second movement of the sequence had a mean latency of $760 \mathrm{msec}(S E=45.7 \mathrm{msec})$, also measured from (auditory) cue onset.

Because the task-irrelevant dot probe served as a measure of the deployment of attention in the visual field, it should not affect the motor task. Specifically, it is important to ascertain that the appearance of the probe at a certain position did not hamper or delay the movement that was about to be programmed. Therefore, we analyzed whether the movement latencies and/ or the spatial accuracy at the first goal were dependent on the factor probe position. For instance, it may be interesting to test whether the landing positions at the 
first goal were slightly shifted towards the probe stimulation. An one-way ANOVA yielded no significant main effects of the factor probe position (with the levels "1st MT," "2nd MT," or "irr") on the latency of movement onset $[F(2,16)=0.711, p>.50]$. Moreover, another ANOVA did not reveal any significant effect of the probe position (with the factor levels "1st MT," "clockwise next position," and "counterclockwise next position") on the directional error at the first goal $[F(2$, $16)=0.006, p>50]$. Therefore, neither the latency of the sequence initialization nor the spatial accuracy at the first goal was systematically affected by where the dot probe appeared in relation to the movement targets. This indicates that the movement task was performed without specific spatial interference from the flashed dot probes.

\section{Event-related Potentials}

In order to determine the distribution of visual attention during the preparation of movement sequences, we analyzed the ERPs that were triggered by the presentation of the dot probe shortly before sequence initialization. The evoked ERPs were collapsed across the four quadrants in which the dot probe could be flashed, and were analyzed with regard to the relative position of the eliciting dot-probe stimulus in relation to the movement goals (factor probe position with the levels: "1st MT," "2nd MT," "irr").

Figure 3 shows the ERPs, which were evoked at the occipital electrode $\mathrm{Oz}$ by dot probes, presented at the various relative stimulus locations. The solid line in the graph represents the ERP in response to dot probes that were flashed at the first movement goal; the dashed line shows the grand averages to dot probes presented at the second movement goal of the pointing sequence. The dotted line finally shows the ERPs to dot probes presented at the third, movement-irrelevant position of the particular trials. The ERPs were characterized by $\mathrm{P} 1$ and N1 components in response to the appearance of the dot probe. Because the dot probe was always presented 150 msec (SOA) after the acoustic go-signal (the onset of the dot probe is marked by the arrow in Figure 3), the evoked P1 peaked at $270 \mathrm{msec}$ (i.e., $120 \mathrm{msec}$ after onset of the dot probe) and the N1 peaked at $320 \mathrm{msec}$. The data show that the amplitude of the N1 component was enhanced if the dot probe was flashed at either the first or second goal location that was relevant for the double-pointing task. In contrast, dot probes at the movement-irrelevant position elicited smaller components.

The enlargement of components evoked by dot probes at movement-relevant locations was confirmed by further statistical analyses. In order to quantitatively compare the $\mathrm{N} 1$ amplitudes depending on the relative dot-probe positions, we extracted the mean voltage in the time window 305 to $335 \mathrm{msec}$ (peak of the N1 com-

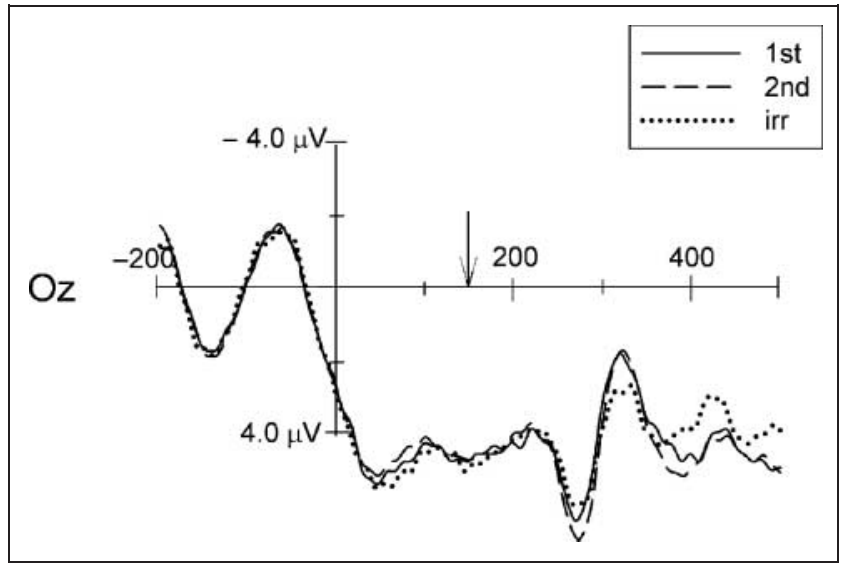

Figure 3. The grand-averaged ERPs evoked at an occipital site $(\mathrm{Oz})$ by the presentation of dot probes. An interval ranging from $200 \mathrm{msec}$ before to $500 \mathrm{msec}$ after the onset of the go-signal is shown. The dot probe could be flashed at either of three relative positions: either at the first movement target ("1st MT," solid line), or the second movement target ("2nd MT," dashed line), or at a third, movement-irrelevant position ("irr," dotted line). Waveforms are collapsed across trials with different absolute positions of the dot probe. The vertical arrow represents the onset of dot probe, which was presented with a constant SOA of $150 \mathrm{msec}$ after the go-signal.

ponent at $320 \pm 15 \mathrm{msec}$ ). First, we computed a twoway ANOVA with the first factor probe position with the levels "1st MT," "2nd MT," and "irrelevant." The other factor coded electrode sites and had the levels "O1," "O2," "P3," "P4," "C3," and "C4." The ANOVA revealed a significant main effect of probe position on the amplitude of the $\mathrm{N} 1$ component $[F(2,16)=6.76$, $p<.01$ ], but no significant main effect of the factor electrode site $[F(5,40)=1.61, p>.18]$. There was a significant interaction between probe position and electrode site $[F(10,80)=3.39, p<.01]$, which is likely due to the fact that the N1 component-and so its modulation-was much less pronounced at more anterior electrode sites. Therefore, we will not interpret the interaction functionally.

We conducted further analyses in order to determine which of the three relative probe positions ("1st MT," "2nd MT," "irr") differed from the other (according to, e.g., Luck, 2005). For this purpose, we ran additional two-way ANOVAs on subsets of the data that included only pairs of relative dot-probe positions (i.e., "1st" vs. "irr," "2nd" vs. "irr," and "1st" vs. "2nd"). The second factor was always "electrode site" with the levels "O1," "O2," "P3," "P4," "C3," and "C4." The amplitude of the N1 component was significantly larger for both the first and second movement goals compared to when the probe appeared at the irrelevant location $[F(1,8)=7.2$, $p<.03$ and $F(1,8)=8.82, p<.02$, respectively]. There was no significant difference, however, between the amplitudes of N1 components that were evoked by probes at the first goal and those in response to probes at the second goal $[F(1,8)=1.3, p>.29]$. 


\section{EXPERIMENT 2}

\section{Methods}

\section{Participants, Stimuli, and Procedure}

Nine students, aged between 23 and 28 years ( 4 men), participated in this second experiment. All had normal vision and were right-handed. The same setup as in the previous experiment was used. We adjusted the configuration of the precue and added intermediate nontarget positions in between the potential movement goals. Figure $1 \mathrm{~B}$ provides a sketch of the modified stimulus sequence.

The initial stimulus configuration now consisted of five crosses that were arranged on a virtual semicircle, which was oriented toward one corner of the screen. All stimuli had the same eccentricity $\left(5^{\circ}\right)$ from the central fixation cross. The participants were asked to perform rapid double-pointing sequences to two out of the five crosses of the configuration as soon as the go-signal was presented.

The first reach had to be directed to the central cross of the configuration. Depending on the frequency of the acoustic go-signal (low- vs. high-frequency beep), participants had to move on either in a clockwise or in a counterclockwise direction to the next-but-one position. A task-irrelevant dot probe was flashed in each trial, 150 msec after the acoustic go-signal. The dot probe was flashed at either the first (condition "1st MT") or the second movement goal (condition "2nd MT") of the particular trial, or at the third-movement-irrelevantposition (condition "irr") that was at the opposite end of the configuration. Additionally, the probe could now also appear at the intermediate position right between the first and second movement goal of that trial (condition "inter").

\section{Design}

Each participant performed five experimental blocks. Each block consisted of 160 trials. The factor probe position now had the four levels "1st MT," "2nd MT," "irr," and "inter." There were four possible cross configurations, corresponding to the four quadrants of the screen. There were two types of acoustical go-signals instructing either clockwise or counterclockwise direction for the second movement part. In total, this led to 32 different conditions ( 4 possible cross configurations $\times$ 2 acoustical direction cues $\times 4$ relative dot-probe positions). The conditions were selected at random in each trial.

\section{ERP Recordings}

The recording parameters were the same as in Experiment 1. Again, the ERP responses were averaged depending on the relative position of the dot probe, irrespective of the quadrant, in which the dot probe may have appeared. The results of this procedure were separate ERP averages for the four relative positions of the dot probe with respect to the actual pointing goals. Averages were computed relative to the 200-msec baseline before the onset of the go-signal. The statistical analysis was based on the mean amplitude of the N1 component elicited by the onset of the dot probe.

\section{Results}

\section{Movement Performance}

$0.8 \%$ of trials had to be discarded because the movement latencies were shorter than $220 \mathrm{msec}$. Another $5.5 \%$ of the trials were rejected because the movement latency was more than 600 msec. In 4.9\%, the first or second movement of the sequence missed the respective target by more than $3^{\circ}$ and these trials were also discarded. The pointing movements were started after $388 \mathrm{msec}$ on average $(S E=24.0 \mathrm{msec})$. The second movement of the sequence had a mean latency of $765 \mathrm{msec}(S E=$ $47.3 \mathrm{msec})$, measured from auditory cue onset.

\section{Event-related Potentials}

The evoked potentials were averaged for each of the four possible relative positions of the dot probe. Figure $4 \mathrm{~A}$ shows the ERPs evoked by the task-irrelevant visual probe stimuli at an occipital electrode site. As in Experiment 1, the dot probe was presented after an SOA of $150 \mathrm{msec}$ after the go-signal. We averaged the evoked ERPs locked in time to the onset of the go-signal.

The solid line shows the ERP in response to dot probes that were flashed at the first movement goal; the long-dashed line shows the grand averages to dot probes presented at the second movement goal of the pointing sequence. The dotted line corresponds to the ERPs to dot probes at the third movement-irrelevant position of the particular trials. The short-dashed line, finally, shows the ERP elicited by probes at the intermediate position. Most interestingly, for the purpose of this second experiment, also dot probes that were flashed at the intermediate positions right between the first and the second reach goals evoked only a small N1 component (short-dashed line). The results resemble the observation of Experiment 1 in that the amplitudes of the N1 were enhanced if the dot probe was flashed at a location that was relevant for the double-pointing task (first and second movement goals). Importantly, dot probes at positions that were not a goal for the planned movement elicited smaller N1 components, although they were flashed at the location between both reach targets (see also Figure 4B).

The N1 amplitudes were compared by extracting the mean amplitude voltage in a 30-msec time window around the peak of the N1 at 320 msec. A two-way ANOVA with the factors probe position (levels "1st MT," 


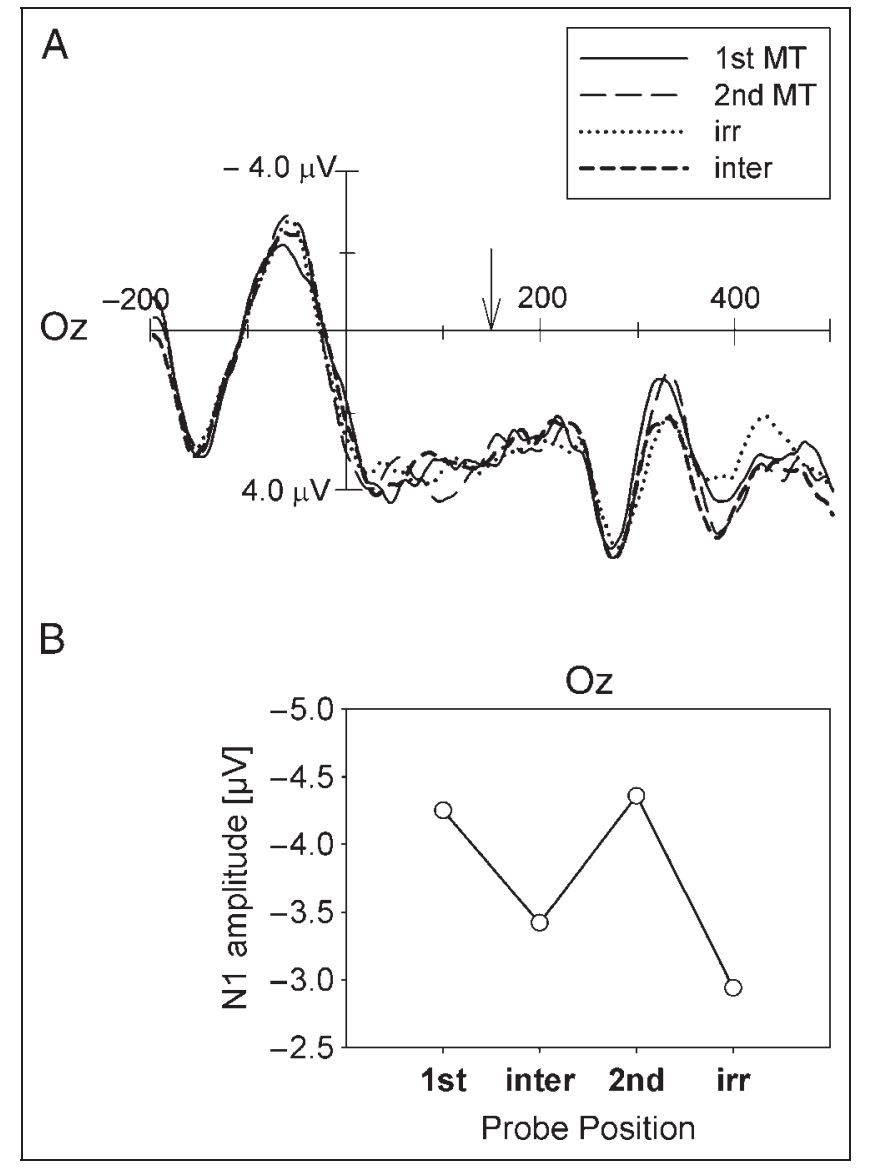

Figure 4. (A) Grand-averaged ERPs at an occipital site evoked by dot probes. The dot probe could be flashed at either of four positions relative to the goal arrangement of the movement task: either at the first ("1st MT," solid line) or the second movement target ("2nd MT," dashed line) or at a third, movement-irrelevant position ("irr," dotted line) or at the intermediate position between both movement goals ("inter," long-dashed line). The vertical arrow shows the onset of the dot probe. (B) The peak-to-peak amplitude of the visual N1 as a function of the relative position of the dot probe.

"2nd MT," "irr," "inter") and the factor electrode site (levels "O1," "O2," "PO7," "PO8," "P5," "P6," "C5," and "C6") revealed a significant main effect of the probe position on the amplitude of the $\mathrm{N} 1$ component $[F(3$, $24)=5.47, p<.004]$, but no significant main effect of the factor electrode site $[F(7,56)=0.74, p>.63]$. Additional ANOVAs were based on subsets of the data including only pairs of relative probe positions (i.e., "1st" vs. "2nd," "1st" vs. "irr," "1st" vs. "inter," "2nd" vs. "irr," "2nd" vs. "inter," and "irr" vs. "inter"). The second factor was always electrode site with the levels "O1," "O2," "PO7," "PO8," "P5," "P6," "C5," and "C6." The most important outcome of this second experiment is that dot probes at the first or second goal elicited significantly higher $\mathrm{N} 1$ amplitudes than probes at the intermediate position $[F(1,8)=5.91, p<.04$ and $F(1,8)=$ $6.38, p<.035$, respectively]. As in Experiment 1, probes at the first and second goals elicited bigger N1 components as probes at the irrelevant position $[F(1,8)=10.0$, $p<.02$ and $F(1,8)=6.39, p<.03$, respectively; see Figure $4 \mathrm{~B}]$. There were no significant differences between components in response to probes at the first versus second goal $[F(1,8)=0.03, p>.8]$, nor between components that were evoked by probes at the irrelevant versus intermediate location $[F(1,8)=0.89, p>$.3] .

\section{DISCUSSION}

\section{Visual Selection of Multiple Movement Goals}

In this study, we used a dot-probe paradigm (see also, e.g., Eimer, Forster, Van Velzen, \& Prabhu, 2005; Heinze et al., 1994; Mangun \& Hillyard, 1988, 1991) in order to test where in the visual field attention is deployed while sequences of manual pointing movements are prepared. For this purpose, a visual probe was presented briefly after a go-signal for the movement sequence but well before the movement initialization, at one of several positions. In each trial, the transient could be presented either at the instructed first or second movement goal, or at positions that were movement-irrelevant in that particular trial. The results show that the N1 components, which were elicited at occipital and parietal sites by the dot probes, differed depending on their relative positions with respect to the motor task goals. Dot probes that were presented at the first or the second movement goal elicited N1 components with higher amplitudes compared to dot probes that appeared at movement-irrelevant locations. We interpret these enlarged components that occurred in response to probes at goal positions as enhanced visual processing and attribute this enhancement to selection-for-action by visual attention.

The dot-probe paradigm has been used in several EEG studies to determine the distribution of attention in the visual field. It is well known that N1 components evoked by dot probes similar to the one used in the present study are modulated by visual attention (e.g., Eimer et al., 2005; Hopf \& Mangun, 2000; Mangun, 1995; Mangun \& Hillyard, 1991). The present study provides the first electrophysiological evidence for the assumption that during the preparation of pointing sequences multiple goal positions of the planned movement sequence are attended in advance to the sequence initialization.

This is in line with our previous behavioral study on the selection of multiple goal positions during the preparation of fast hand movement sequences (Baldauf et al., 2006), where we showed that during sequence preparation participants could better identify target letters at all goal locations of a planned sequence than at movementirrelevant positions. We also showed that more attention is allocated to the first reach goal than to subsequent, and that intermediate positions that lie between two reaching targets are not selected. Interestingly, the N1 components that we recorded in the present experiments in response to dot probes flashed at the first and second movement goals did not differ from each other. 
This suggests that the first and the second movement goals are attended to about the same degree, which is in some contrast to our behavioral findings. One possible explanation for these differing results may be that the observed modulation of the N1 component did not result from an enhancement of those amplitudes that were evoked by probe stimuli at the movement goals, but rather resulted from a decrease of the amplitude in response to probes at movement-irrelevant locations. Following this alternative view, the go-signal in Experiment 1 may indicate that one of the potential goals, namely, the irrelevant location, has to be ignored and attention may not be allocated toward the movement goals but shifted away from the position that is no longer relevant for the upcoming movement.

Another possibility that should be considered is that the attentional activations of the first and second movement goals could have different time courses. For example, it could be the case that the impending reach goal is activated first and briefly after that activation raises also for the subsequent movement goal. Because we only sampled the deployment of attention at $150 \mathrm{msec}$ after the go-signal, we cannot rule out this alternative.

\section{Relation to Other Recent ERP Studies on Movement Preparation}

Other recent EEG studies have also investigated the relationship between covert shifts of attention and manual response preparation. Eimer et al. (2005), for example, conducted an ERP study to explore whether shifts of somatosensory attention are triggered when unimanual responses are prepared. In their task, subjects had to prepare to lift either their right or left index finger after a certain delay. The authors observed enhanced somatosensory ERP components when during a delay period task-irrelevant tactile probes were delivered to the cued hand. Analog results were reported by further studies using similar approaches (e.g., Eimer, Van Velzen, \& Driver, 2002; Van der Lubbe et al., 2000). These findings were interpreted as covert shifts of visual attention as a consequence of movement preparation. However, all these studies investigated manual response tasks that did not involve actual goal-directed reach movements to certain target positions but mere effector selections (e.g., lifting the left vs. right index finger). They provide some evidence that the preparation of a motor response with either the left or right hand causes attention to be shifted to the chosen effector side. In contrast, our results show that attention-for-action shifts to target locations of visually guided reaches, similar to what has been previously observed in the programming of single saccades (see e.g., Eimer, Van Velzen, Gherri, \& Press, 2006; Van der Stigchel \& Theeuwes, 2005). Furthermore, most physiological studies on the preparation of reaching movements measured preparation activity during an instructed delay before the go-signal. In contrast, we mea- sured the deployment of visual attention to reach goals shortly after the go-signal, before the reach sequence started.

\section{Spatially Distinct Foci of Attention}

In our second experiment, we presented the dot probe also at an irrelevant intermediate position between the first and the second goal. By this we wanted to analyze whether the participants attended to an extended region of space if they are requested to perform speeded actions to multiple movement goals. The results of Experiment 1 could be explained by a possible widening of the attentional focus as to cover both target positions simultaneously (Eriksen \& James, 1986; Eriksen \& Yeh, 1985). The results of Experiment 2, however, showed that the N1 component evoked by dot probes at intervening locations was not enhanced in comparison to other movement-irrelevant positions. It might be argued that in Experiment 2 the reach goals and the intermediate position are not arranged linearly but in a semicircular way. Hence, there remains the possibility that the focus of attention is continuous and stretches across both reach goals as a very narrow and linear band lying a bit laterally to the irrelevant, intermediate position. However, this alternative does not seem likely because the dot probes presented at the intermediate position considerably overlap with any subspace connecting both movement targets (see Figure 1B). From our data, we suggest that the parallel selection of multiple reach goals also implies a splitting of the attentional beam into noncontiguous foci.

These results are in line with previous findings in sequential movement production (Baldauf \& Deubel, 2008a; Baldauf et al., 2006; Godijn \& Theeuwes, 2003) showing that the discrimination performance at goal positions is superior as compared to irrelevant and intervening positions. The results are evidence for the assumption that several movement-relevant goals are attended by spatially distinct foci, whereas intermediate, movement-irrelevant locations are not selected. The parallel selection of several parts of the visual field during movement preparation may be spatially rather accurate and specifically restricted just to certain objects of relevance. In our experimental design, the intermediate positions between the two movement goals were nontargets in the sense that they had to be avoided during the sequence production. Such nontargets may remain unselected because they are at a higher risk of interfering with the action goals.

Interestingly, our findings are not consistent with previous results by Heinze et al. (1994), who suggested that attention cannot be divided into multiple distinct foci. In their ERP study, they instructed the participants to covertly attend to two out of four locations and to compare visual stimuli that were presented at these positions. In a blocked design, the two attended positions 
could be either adjacent to each other or separated by an intervening position that had not been attended. In rare interleaving trials, a dot probe was presented at any of the four positions. The N1 component evoked by these visual transients differed in dependence of whether or not the location where it was presented had to be attended. However, if the dot probe appeared at an irrelevant intervening position right between the two locations that had to be attended the evoked sensory response was not suppressed but was equally enhanced. This suggests that attention formed a unitary region of space that may be adjusted as to cover multiple locations of interest, according to the task demands. One possible explanation of the differing results regarding the selection of intervening locations might be that only during the preparation of movements the required selection of goal positions is more specific and accurately bound to goal objects. It may be speculated that the preparation of precise pointing movements poses higher demands on the spatial resolution of the involved selection processes as compared to instructed attention shifts in mere perceptual tasks (as in the task of Heinze et al.).

\section{Neural Correlates}

In this study, we found that early ERP components in response to visual transients were modulated by the preparation of goal-directed hand movements. The modulation of the evoked N1 components most likely arose from enhanced processing of movement-relevant information in early visual areas. Therefore, we propose that this modulation reflects the result of attentional top-down signals, that selectively bias early stages of visual processing, rather than representing brain activity involved in the movement programming, which provides the source for such attentional signals.

The obvious question arises where in the brain the top-down signals may originate. Several cortical structures are possible candidates to provide such attentional top-down signals for sequential movement preparation. Motor-related structures in the frontal cortex are known to be involved in the long-term storage of multiple movement parts as well as in the crucial temporal ordering of various movement components during sequence planning (Averbeck \& Lee, 2007; Ninokura, Mushiake, \& Tanji, 2003, 2004; Ohbayashi, Ohki, \& Miyashita, 2003; Averbeck, Chafee, Crowe, \& Georgopoulos, 2002). Mushiake, Saito, Sakamoto, Itoyama, and Tanji (2006) recorded from neurons in the lateral prefrontal cortices (PFC) of monkeys that navigated by stepwise cursor movements through a maze on the screen. During the movement preparation, various PFC neurons encoded the directions of all forthcoming cursor movements, even if there were considerable delays between all movement steps. Also, the supplementary motor area and the premotor area are supposed to code for several movements ahead (Shima \& Tanji, 2000; Tanji \& Shima, 1994). Nevertheless, one may argue that many of these frontal structures are unlikely to directly provide attentional signals to visual areas because many of them encode movement directions with respect to the actual hand position rather than coding for goal locations in eye-centered coordinates. In the dorsal part of the premotor area, for example, neurons encode reach goals with respect to the eye and hand (Pesaran, Nelson, \& Andersen, 2006). Representations in such complex, noneye-centered frames of reference would first require back-transforming the information in order to facilitate perception at certain positions that are retinotopically coded in early visual areas.

On the other hand, the posterior regions of the parietal cortex (PPC) are known to play an important role in shifting spatial attention. In humans, lesions to the PPC cause disorders in the representation of space (e.g., neglect). Also, the planning and execution of goaldirected movements are often affected by injuries of the PPC (e.g., limb apraxia and optic ataxia; see Balint, 1909). More specifically, in monkey PPC, several substructures have been identified that provide multiple representations of space for different kinds of actions (e.g., Snyder et al., 2000; Andersen et al., 1997). Although the lateral intraparietal area, for example, is crucial for the generation of saccadic eye movements, the nearby parietal reach region (PRR) shows spatially selective activation before reaching movements. Andersen and colleagues interpreted the activity of PRR neurons as the encoding of reaching intentions (Buneo \& Andersen, 2006; Snyder, Batista, \& Andersen, 1997). In general, the effector-specific and eye-centered neuronal coding in the PRR would be the most likely source for attentional signals that could facilitate visual perception at the reach goal via feedback loops. Indeed, in a recent study, Baldauf, Cui, and Andersen (2007) recorded from single neurons in the PRR of two monkeys while the animals were preparing for a double reach task to two goal locations. They first found evidence that neuronal populations in the PRR encode in parallel multiple movement goals of a planned hand movement sequence. This eye-centered planning activity in the parietal cortex may be the source of attentional top-down signals that directly cause enhanced visual processing of multiple goal positions.

Our study provides first physiological evidence for covert attention shifts to multiple reach goals of a planned double-step pointing sequence. The data show that, before the sequence starts, early stages of visual processing are enhanced at the immediate as well as at a subsequent goal position.

\section{Acknowledgments}

This research was funded by the Deutsche Forschungsgemeinschaft (Graduate Programme GRK 1091 and Research Group 
De336/2) and by the Cluster of Excellence "Cognition in Technical Systems." We thank Klaus Gramann for his technical support and for discussions at an early stage of this project, and Rachel Berquist for her valuable comments on the manuscript.

Reprint requests should be sent to Daniel Baldauf, Department Psychologie, Ludwig-Maximilians-Universität München, Leopoldstrasse 13, 80802 Munich, or via e-mail: baldauf@psy. uni-muenchen.de.

\section{REFERENCES}

Allport, D. A. (1987). Selection for action: Some behavioral and neurophysiological considerations of attention and action. In H. Heuer \& A. F. Sanders (Eds.), Perspectives on perception and action (pp. 395-419). Hillsdale, NJ: Erlbaum.

Andersen, R. A., \& Buneo, C. A. (2002). Intentional maps in posterior parietal cortex. Annual Review of Neuroscience, 25, 189-220.

Andersen, R. A., Snyder, L. H., Bradley, D. C., \& Xing, J. (1997). Multimodal representation of space in the posterior parietal cortex and its use in planning movements. Annual Review of Neuroscience, 20, 303-330.

Averbeck, B. B., Chafee, M. V., Crowe, D. A., \& Georgopoulos, A. P. (2002). Parallel processing of serial movements in prefrontal cortex. Proceedings of the National Academy of Sciences, U.S.A., 99, 13172-13177.

Averbeck, B. B., \& Lee, D. (2007). Prefrontal neural correlates of memory for sequences. Journal of Neuroscience, 27, 2204-2211.

Awh, E., Armstrong, K. M., \& Moore, T. (2006). Visual and oculomotor selection: Links, causes and implications for spatial attention. Trends in Cognitive Sciences, 10, 124-130.

Baldauf, D., Cui, H., \& Andersen, R. A. (2007). The posterior parietal cortex encodes the first and second goal in sequential reaching arm movements. Annual meeting of the Society for Neuroscience, 2007. Program no. 397.3. San Diego, CA.

Baldauf, D., \& Deubel, H. (2008a). Properties of attentional selection during the preparation of sequential saccades. Experimental Brain Research, 184, 411-425.

Baldauf, D., \& Deubel, H. (2008b). Visual attention during the preparation of bimanual movements. Vision Research, 48, 549-563.

Baldauf, D., Wolf, M., \& Deubel, H. (2006). Deployment of visual attention before sequences of goal-directed hand movements. Vision Research, 46, 4355-4374.

Balint, R. (1909). Seelenlähmung des Schauens, optische Apraxie, räumliche Störung der Aufmerksamkeit.

Monatsschriften für Psychiatrische Neurologie, 25, 51-81.

Ballard, D. H., Hayhoe, M. M., \& Pelz, J. B. (1995). Memory representations in natural tasks. Journal of Cognitive Neuroscience, 7, 66-80.

Buneo, C., \& Andersen, R. A. (2006). The posterior parietal cortex: Sensorimotor interface for the planning and online control of visually guided movements. Neuropsychologia, 44, 2594-2606.

Colby, C. L. (1998). Action-oriented spatial reference frames in cortex. Neuron, 20, 15-24.

Deubel, H., \& Schneider, W. X. (1996). Saccade target selection and object recognition-Evidence for a common attentional mechanism. Vision Research, 36, 1827-1837.

Deubel, H., Schneider, W. X., \& Paprotta, I. (1998). Selective dorsal and ventral processing: Evidence for a common attentional mechanism in reaching and perception. Visual Cognition, 5, 81-107.
Eimer, M., Forster, B., Van Velzen, J., \& Prabhu, G. (2005). Covert manual response preparation triggers attentional shifts: ERP evidence for the premotor theory of attention. Neuropsychologia, 43, 957-966.

Eimer, M., Van Velzen, J., \& Driver, J. (2002). Crossmodal interactions between audition, touch and vision in endogenous spatial attention: ERP evidence on preparatory states and sensory modulations. Journal of Cognitive Neuroscience, 14, 254-271.

Eimer, M., Van Velzen, J., Gherri, E., \& Press, C. (2006). Manual response preparation and saccade programming are linked to attention shifts: ERP evidence for covert attentional orienting and spatially specific modulations of visual processing. Brain Research, 1105, 7-19.

Eriksen, C. W., \& James, J. D. (1986). Visual attention within and around focal attention: A zoom lens model. Perception E Psychophysics, 40, 225-240.

Eriksen, C. W., \& Yeh, Y. Y. (1985). Allocation of attention in the visual field. Journal of Experimental Psychology: Human Perception and Performance, 11, 583-597.

Godijn, R., \& Theeuwes, J. (2003). Parallel allocation of attention prior to the execution of saccade sequences. Journal of Experimental Psychology: Human Perception and Performance, 29, 882-896.

Gratton, G., Coles, M. G. H., \& Donchin, E. (1983). A new method for off-line removal of ocular artifact. Electroencephalography and Clinical Neurophysiology, 55, 468-484.

Hayhoe, M., Shrivastava, A., Mruczek, R., \& Pelz, J. B. (2003). Visual memory and motor planning in natural tasks. Journal of Vision, 3, 49-63.

Heinze, H. J., Luck, S. J., Munte, T. F., Gös, A., Mangun, G. R., \& Hillyard, S. A. (1994). Attention to adjacent and separate positions in space: An electrophysiological analysis. Perception E Psychophysics, 56, 42-52.

Hoffman, J. E., \& Subramaniam, B. (1995). The role of visual attention in saccadic eye movements. Perception $\mathcal{E}$ Psychophysics, 57, 787-795.

Hopf, J. M., \& Mangun, G. R. (2000). Shifting visual attention in space: An electrophysiological analysis using high spatial resolution mapping. Clinical Neurophysiology, 111, $1241-1257$.

Ihaka, R., \& Gentleman, R. (1996). R: A language for data analysis and graphics. Journal of Computational and Graphical Statistics, 5, 299-314.

Irwin, D. E., \& Gordon, R. D. (1998). Eye movements, attention, and transsaccadic memory. Visual Cognition, 5, $127-155$.

Jeannerod, M. (1994). The representing brain: Neural correlates of motor intention and imagery. Behavioral and Brain Sciences, 17, 187-245.

Kawashima, R., Naitoh, E., Matsumura, M., Itoh, H., Ono, S., \& Satoh, K. (1996). Topographic representation in human intraparietal sulcus of reaching and saccade. NeuroReport, 7 , 1253-1256.

Kowler, E., Anderson, E., Dosher, B., \& Blaser, E. (1995). The role of attention in the programming of saccades. Vision Research, 35, 1897-1916.

Luck, S. J. (2005). An introduction to the event-related potential technique. Cambridge, MA: MIT Press.

Luck, S. J., \& Hillyard, S. A. (1995). The role of attention in feature detection and conjunction discrimination: An electrophysiological analysis. International Journal of Neuroscience, 80, 281-297.

Luck, S. J., Hillyard, S. A., Mouloua, M., Woldorff, M. G., Clark, V. P., \& Hawkins, H. L. (1994). Effects of spatial cuing on luminance detectability: Psychophysical and 
electrophysiological evidence for early selection. Journal of Experimental Psychology: Human Perception and Performance, 20, 887-904.

Mangun, G. R. (1995). Neural mechanisms of visual selective attention. Psychophysiology, 32, 4-18.

Mangun, G. R., \& Hillyard, S. A. (1988). Spatial gradients of visual attention: Behavioural and electrophysiological evidence. Electroencephalography and Clinical Neurophysiology, 70, 417-428.

Mangun, G. R., \& Hillyard, S. A. (1990). Allocation of visual attention to spatial locations. Trade-off functions of event-related brain potentials and detection performance. Perception E Psychophysics, 47, 532-550.

Mangun, G. R., \& Hillyard, S. A. (1991). Modulations of sensory-evoked brain potentials indicate changes in perceptual processing during visual-spatial priming. Journal of Experimental Psychology: Human Perception and Performance, 17, 1057-1074.

Mangun, G. R., Hillyard, S. A., \& Luck, S. (1993). Electrocortical substrates of visual selective attention. In D. Meyer \& S. Kornblum (Eds.), Attention and performance XIV (pp. 219-243). Cambridge, MA: MIT Press.

Mennie, N., Hayhoe, M., \& Sullivan, B. (2007). Look-ahead fixations: Anticipatory eye movements in natural tasks. Experimental Brain Research, 179, 427-442.

Mushiake, H., Saito, N., Sakamoto, K., Itoyama, Y., \& Tanji, J. (2006). Activity in the lateral prefrontal cortex reflects multiple steps of future events in action plans. Neuron, 50, 631-641.

Neumann, O. (1987). Beyond capacity: A functional view of attention. In H. Heuer \& A. F. Sanders (Eds.), Perspectives on perception and action (pp. 361-394). Hillsdale, NJ: Erlbaum.

Ninokura, Y., Mushiake, H., \& Tanji, J. (2003). Representation of the temporal order of visual objects in the primate lateral prefrontal cortex. Journal of Neurophysiology, 89, $2868-2873$.

Ninokura, Y., Mushiake, H., \& Tanji, J. (2004). Integration of temporal order and object information in the monkey lateral prefrontal cortex. Journal of Neurophysiology, 91, $555-560$.

Ohbayashi, M., Ohki, K., \& Miyashita, Y. (2003). Conversion of working memory to motor sequences in the monkey premotor cortex. Science, 301, 233-236.

Pelz, J. B., \& Canosa, R. (2001). The coordination of eye, head, and hand movements in a natural task. Experimental Brain Research, 139, 266-277.

Pesaran, B., Nelson, M. J., \& Andersen, R. A. (2006). Dorsal premotor neurons encode the relative position of the hand, eye and goal during reach planning. Neuron, 51, 125-134

Posner, M. I. (1980). Orienting of attention. Quarterly Journal of Experimental Psychology, 32, 3-25.

Rizzolatti, G., Riggio, L., \& Sheliga, B. M. (1994). Space and selective attention. In C. Umilta \& M. Moscovitch (Eds.), Attention and performance XV. Conscious and nonconscious information processing (pp. 231-265). Cambridge: MIT Press.

Schneider, W. X., \& Deubel, H. (2002). Selection-for-perception and selection-for-spatial motor- action are coupled by visual attention: A review of recent findings and new evidence from stimulus-driven saccade control. In W. Prinz \& B. Hommel (Eds.), Attention and performance: XIX. Common mechanisms in perception and action (pp. 609-627). Oxford: Oxford University Press.

Shima, K., \& Tanji, J. (2000). Neuronal activity in the supplementary and pre-supplementary areas for temporal organization of multiple movements. Journal of Neurophysiology, 84, 2148-2160.

Snyder, L. H., Batista, A. P., \& Andersen, R. A. (1997). Coding of intention on the posterior parietal cortex. Nature, 386, $167-170$.

Snyder, L. H., Batista, A. P., \& Andersen, R. A. (2000). Intention-related activity in the posterior parietal cortex. Vision Research, 40, 1433-1441.

Tanji, J., \& Shima, K. (1994). Role for supplementary motor area cells in planning several movements ahead. Nature, 371, 413-416.

Treisman, A., \& Gelade, G. (1980). A feature-integration theory of attention. Cognitive Psychology, 12, 97-136.

Van der Lubbe, R. H. J., Wauschkuhn, B., Wascher, E., Niehoff, T., Kömpf, D., \& Verleger, R. (2000). Lateralized EEG components with direction information for the preparation of saccades versus finger movements. Experimental Brain Research, 132, 163-178.

Van der Stigchel, S., \& Theeuwes, J. (2005). The influence of attending to multiple locations on eye movements. Vision Research, 45, 1921-1927.

Wolf, W., \& Deubel, H. (1997). P31 phosphor persistence at photopic luminance level. Spatial Vision, 4, 323-333. 